\title{
Chiral Schwinger model with Faddeevian anomaly and its BRST quantization
}

\author{
Sanjib Ghosal, Anisur Rahaman ${ }^{\mathrm{a}}$ \\ Hooghly Mohsin College, Chinsurah, Hooghly, West Bengal 712101, India
}

Received: 3 October 2019 / Accepted: 7 January 2020 / Published online: 1 February 2020

(C) The Author(s) 2020

\begin{abstract}
We consider chiral Schwinger model with Faddeevian anomaly, and carry out the quantization of both the gauge-invariant and non-invariant version of this model has been. Theoretical spectra of this model have been determined both in the Lagrangian and Hamiltonian formulation and a necessary correlation between these two are made. BRST quantization using BFV formalism has been executed which shows spontaneous appearance of Wess-Zumino term during the process of quantization. The gauge invariant version of this model in the extended phase space is found to map onto the physical phase space with the appropriate gauge fixing condition.
\end{abstract}

\section{Introduction}

Chiral Schwinger model is an exactly solvable lower dimensional field theoretical model [1-5]. Initially, it was derived from Schwinger model $[6,7]$ replacing the vector interaction of it by by chiral interaction. This chiral generation was initially attempted in the article [8], however it failed to receive much attention because of the non-unitarity associated with it stood as an obstacle in the way to be physically sensible. The long suffering of this model was cured by Jackiw and Rajaraman in their seminal work [1] prescribing a very effective as well as compelling term by hand allowing electromagnetic current to take anomalous nature. It was later shown in [9] that it was the fascinating one loop correction term that entered during regularization. The model then became a fertile field of investigation in the regime of lower dimensional anomalous gauge theory [2,3,5,10-23] and extension of this model started in different direction [1-3,5,9-12] like the extension of its ancestor the celebrated Schwinger model $[7,13,14]$. Plenty of investigations on this model were carried out in connection with the confinement-de-confinement aspect of fermion, renormalization, [1-3,5,9-12] regenera-

a e-mails: anisur.rahman@saha.ac.in; manisurn@gmail.com tion of the lost symmetry due to the compelling electromagnetic anomaly [24-26], BRST invariant reformulation etc. [29-31].

Mitra in his seminal work [5] however showed that this model remained physically sensible in all respect with a very special type of anomaly which was termed by Mitra as Faddeevian anomaly [32-35]. It does not belong to JackiwRajaraman's one parameter class of anomaly. The most surprising aspect of this model with the Faddeevian anomaly is the maintenance of physical Lorentz invariance despite the Lorentz non-covariant structure of this anomaly. Photon here too acquires mass via kind of dynamical symmetry breaking, but unlike the Jackiw-Rajaraman version of chiral Schwinger model the fermion which remains unconfined has a definite chirality. Another attraction of this model is the admissibility of description of this model in terns of chiral boson [36-38] which is the basic ingredient of the heterotic string theory. In fact, it leads to the gauged model of Flurenini-Jackiw type of chiral bosom [26,39].

This model with these two independent physically sensible one loop correction (Counter term) and their ancestor, viz, the celebrated Schwinger model are so fascinating with so many interesting surprise [2,3,5,10-23,26-28] that till now it remains as a field of several investigations in the exactly solvable regime of lower dimensional field theoretical models. This article is an extension of chiral Schwinger model with Faddeevian type of anomaly due to Mitra towards its quantization in both the gauge invariant as well as gauge non-invariant manner along with the BRST invariant reformulation with an attempt to derive the appropriate WessZumino term. How the the exact solution of the gauge invariant version maps onto the solution with the usual gauge noninvariant version has also been explored.

The paper is organized as follows. Sect. 2, contains a brief review of the chiral Schwinger model with Faddeevian anomaly due to Mitra. In Sect. 3, the physical spectrum is determined in the Lagrangian formulation. Section 4 is 
devoted with constrained quantization of this model in a and transparent as well as desired manner such that it enables to make a correlation with the spectrum obtained in Sect. 3 . In Sect. 5 we give a brief introduction of Batalin, Fradkin, Viilcovisky (BFV) formulation. In Sect. 6 we carry out the BRST quantization of this model with BVF formulation with an effort to generate the appropriate Wess-Zumino term. Section 7. deals with quantization of the gauge invariant version of the model. How the gauge invariant model with WessZumino term maps onto the usual gauge invariant version of the model is shown in Sect. 8. Final Sect. 9. holds a brief summary and discussion over the work.

\section{A brief review of the chiral Schwinger model with Mitra type Faddevian anomaly}

Replacing the vector interaction between matter and gauge field of the Schwinger model by chiral interaction the chiral Schwinger model was described by the action

$\mathcal{S}_{C F}=\int d^{2} x\left[i \bar{\psi}\left[\partial+i e A\left(1+\gamma_{5}\right)\right] \psi-\frac{1}{4} F_{\mu \nu} F^{\mu \nu}\right]$.

In $(1+1)$ dimension left and right handed fermionic part can be decoupled and the integration over the right handed fermion of the action (1) contributes a field independent constant term which can be absorbed within the normalization. Therefore the effective action gets simplified to

$\mathcal{S}_{f}=\int d^{2} x\left[i \bar{\psi}_{L}(\partial+i 2 e \sqrt{\pi} A) \Psi_{L}-\frac{1}{4} F_{\mu \nu} F^{\mu \nu}\right]$.

The generating functional of the above theory defined by the action (2) can be expressed as

$Z[A]=\int d \phi e^{i \mathcal{S}_{C B}}$,

where $\mathcal{S}_{C B}$ is the the bosonized version of the action (2):

$\mathcal{S}_{C B}=\int d^{2} x \mathcal{L}_{C H}$,

with

$$
\begin{aligned}
L_{C H}=\int & d x \mathcal{L}_{C H}=\int d x\left[\frac{1}{2} \partial_{\mu} \phi \partial^{\mu} \phi\right. \\
& +e\left(\eta^{\mu \nu}+\epsilon^{\mu \nu}\right) \partial_{\nu} \phi A_{\mu} \\
& \left.+\frac{1}{2} e^{2}\left(A_{0}^{2}-3 A_{1}^{2}-2 A_{1} A_{0}\right)-\frac{1}{4} F_{\mu \nu} F^{\mu \nu}\right] .
\end{aligned}
$$

From the article [5] we have learned that the study of chiral fermion in presence of the obstacle posed by the gravitational anomaly is tractable with the bosonized version and the process of bosonization is crucially tied up with the regularization. When one wants to integrate out the left handed fermion it drives us to incorporate the intriguing one loop correction term in order to get rid of the divergence cropped up during the computation of the fermionic determinant. Different correction terms may get involved depending on the choice of regularization. The matter that has to be taken care of during regularization with a serious measure indeed be the maintenance of physical Lorentz invariance. In this context, we must remember that apparent Lorentz non-covariance not always pose any obstruction in the way of sustaining physical Lorentz invariance $[5,10,11]$. Here we choose the Faddeevian type of anomaly [32,33] which was developed, and studied as well, in the articles $[5,10,11]$. With this Faddeevian anomaly the bosonized action reads

$$
\begin{aligned}
\mathcal{S}_{C B}=\int & d^{2} x\left[\frac{1}{2} \partial_{\mu} \phi \partial^{\mu} \phi+e\left(\eta^{\mu \nu}+\epsilon^{\mu \nu}\right) \partial_{\nu} \phi A_{\mu}\right. \\
& \left.+\frac{1}{2} e^{2}\left(A_{0}^{2}-3 A_{1}^{2}-2 A_{1} A_{0}\right)-\frac{1}{4} F \mu \nu F^{\mu \nu}\right] .
\end{aligned}
$$

The action in Eq. (6) was initially formulated in [5] where the author termed it as chiral Schwinger model with Faddeevian anomaly. Note that there is no Lorentz co-variance at the action level however in due course we will find that this will not in any way hinders the process of preservation of the physical Lorentz invariance of the theory.

The Levicivita symbol in $(1+1)$ dimension is chosen as $\epsilon^{01}=-\epsilon_{01}=-1$ and the Minkowski metric $g^{\mu \nu}=$ diag $(1,-1)$ as usual. The indices $\mu$ and $\nu$ takes the value 0 and 1 in two dimensional space time. The advantage of using the bosonized version is that the anomaly gets subsumed within within the theory during the process of bosonization. So the tree level bosonized theory acquires the effect of one loop correction. In the following section we will proceed to study the theoretical spectrum of this model in Lagrangian formulation.

\section{Solution in the Lagrangian formulation}

To find out the theoretical spectrum we compute the equations of motion of the fields describing the Lagrangian (6). The Euler-Lagrange equations for the field $\phi, A_{1}$ and $A_{0}$ that results from the Lagrangian (6) are

$$
\begin{aligned}
& \square \phi+\left(\eta_{\mu \nu}+\epsilon_{\mu \nu}\right) \partial^{v} A^{\mu}=0, \\
& \ddot{A}_{1}-\dot{A}_{0}^{\prime}+e\left(\dot{\phi}+\phi^{\prime}\right)+e^{2}\left(A_{0}+3 A_{1}\right)=0, \\
& A_{0}^{\prime \prime}-\dot{A}_{1}^{\prime}-e\left(\dot{\phi}+\phi^{\prime}\right)-e^{2}\left(A_{0}-A_{1}\right)=0 .
\end{aligned}
$$

To simplify the above equations we introduce the following ansatz for the field $A_{\mu}$ and $\phi$ :

$$
\begin{aligned}
& A_{\mu}=\frac{1}{2 e^{2}}\left[\epsilon_{\mu \nu} \partial^{\nu} \mathcal{F}+\epsilon_{\mu \nu} \partial^{\nu} \mathcal{H}\right], \\
& \phi=\frac{1}{2 e} \mathcal{F}+\mathcal{H} .
\end{aligned}
$$


A straightforward calculation leads us to the following two decoupled equations of motion.

$\left[\square+4 e^{2}\right] \square \mathcal{F}=0$,

$\partial_{+} \mathcal{H}=0$.

Here $\partial_{+}=\partial_{0}+\partial_{1}$. So it indicates that the theoretical spectrum contains a massive boson with mass $2 e$ and a massless chiral boson. Note that $\pi_{1}=-\epsilon_{\mu \nu} \partial^{\nu \mu}=\square F$. To correlate these fields to the scalar and gauge fields describing the Lagrangian (1) we need to consider the solution of this model in the Hamiltonian formulation.

\section{Solution in the Hamiltonian formulation}

We now turn towards the Hamiltonian analysis of the theory in order to obtain the theoretical spectrum in a transparent manner which will enable us to to correlate the fields $\mathcal{F}, \mathcal{H}$ to the fields used in the description of the Lagrangian. We should mention that the Hamiltonian analysis was already done in [5]. We will, in fact, describe it in more detail and transparent manner which will help us to make the necessary correlation. It will indeed make this article self contained. We will proceed to find out the theoretical spectra of this model in the Hamiltonian formulation which necessities to pursue with the constraint analysis of this theory. From the standard definition the canonical momenta corresponding to the matter field $\phi$, the gauge field components $A_{0}$ and $A_{1}$ are found out.

$$
\begin{aligned}
& \frac{\partial \mathcal{L}_{C H}}{\partial \dot{\phi}}=\pi_{\phi}=\dot{\phi}+e\left(A_{0}-A_{1}\right), \\
& \frac{\partial \mathcal{L}_{C H}}{\partial \dot{A}_{1}}=\pi_{1}=\left(\dot{A}_{1}-A_{0}^{\prime}\right), \\
& \frac{\partial \mathcal{L}_{C H}}{\partial \dot{A}_{0}}=\pi_{0} \approx 0 .
\end{aligned}
$$

Here $\pi_{\phi}$ represents the momentum corresponding to the scalar field $\phi$, and $\pi_{1}$ and $\pi_{0}$ stand for the momenta corresponding to gauge fields $A_{0}$ and $A_{1}$. The canonical Hamiltonian of the theory is computed using Eqs. (14), (15) and (16) which reads

$$
\begin{aligned}
H_{C}=\int & d x\left[\frac{1}{2}\left(\pi_{1}^{2}+\pi_{\phi}^{2}+\phi^{\prime 2}\right)\right. \\
& \left.+\pi_{1} A_{0}^{\prime}-e\left(A_{0}-A_{1}\right)\left(\pi_{\phi}+\phi^{\prime}\right)+2 e^{2} A_{1}^{2}\right] .
\end{aligned}
$$

The right hand side of the expression of momentum $\pi_{0} \approx 0$ does not contain any time derivative, so it is the primary constraint of the theory. The preservation of this constraint leads to the secondary constraint:

$G=\pi_{1}^{\prime}+e\left(\pi_{\phi}+\phi^{\prime}\right) \approx 0$, which is generally termed as the Gauss law of the theory. The Lagrange multipliers $u$ and $v$ are having the dimension of velocity.

$H_{E F F}=\int d x\left[\mathcal{H}_{C}+u \pi_{0}+v\left(\pi_{1}^{\prime}+e\left(\pi_{\phi}+\phi^{\prime}\right)\right)\right]$.

Now we can determine the velocity $v$ which comes out to be

$v=\phi-e\left(A_{0}-A_{1}\right)$.

Plugging in the velocity $v$ in (19) the consistency criteria of the constraints lads to the final constraint

$-2 e^{2}\left(A_{1}+A_{0}\right)^{\prime} \approx 0$.

The velocity $u$ gets fixed from the preservation of the final constraint (21):

$\left.u=-\pi_{1}-A_{0}^{\prime}\right)$.

Therefore the theory under consideration contains three constraints in its phase space and these constraints precisely are

$\Omega_{1}=\pi_{0} \approx 0$,

$\Omega_{2}=A_{0}+A_{1} \approx 0$,

$\Omega_{3}=\pi_{1}^{\prime}+e\left(\pi_{\phi}+\phi^{\prime}\right) \approx 0$.

According to the Dirac terminology these constraints are all weak conditions at this stage. It is also found that the Poisson bracket between $G(x)$ and $G(y)$ is

$[G(x), G(y)]=2 e^{2} \delta(x-y)^{\prime}$.

In the chiral Schwinger model [1] this Poisson bracket (26) corresponding to the Gauss law constraint was found to gave vanishing contribution. Faddeev initially noticed that anomaly might make Poisson bracket between $G(x)$ and $G(y)$ nonzero [32,33], and the constraint acquires second class nature and consequently gauge invariance gets lost. He, however, showed that a systematic quantization of the theory would be possible in that situation too, but the system might posses extra degrees of freedom.

This is a system endowed with three constraints in the phase space. So ordinary Poisson bracket becomes inadequate and for the determination of the phase space structure of this system one does need the Dirac bracket [40], which is defined by

$$
\begin{array}{r}
{[A(x), B(y)]^{*}=[A(x), B(y)]-\int\left[A(x), \Omega_{i}(\eta)\right]} \\
C_{i j}^{-1}(\eta, z)\left[\Omega_{j}(z), B(y)\right] d \eta d z,
\end{array}
$$

where $C_{i j}^{-1}(x, y)$ is computed using the following equation

$$
\int C_{i j}^{-1}(x, z)\left[\Omega_{j}(z), \Omega_{k}(y)\right] d z=\delta(x-y) \delta_{i k} .
$$


The system under consideration described by the Lagrangian (5), has the following $C_{i j}(x, y)$ :

$C_{i j}(x, y)=\left(\begin{array}{ccc}0 & -\delta(x-y) & 0 \\ \delta(x-y) & 0 & -\delta^{\prime}(x-y) \\ 0 & -\delta^{\prime}(x-y) & 2 e^{2} \delta^{\prime}(x-y)\end{array}\right)$.

The nonsingular nature of the matrix $C_{i j}$ indicates that by all means it has an inverse which is given by

$$
C_{i j}^{-1}(x, y)=\left(\begin{array}{ccc}
-\frac{1}{2 e^{2}} \delta^{\prime}(x-y) & \delta(x-y) & \frac{1}{2 e^{2}} \delta(x-y) \\
-\delta(x-y) & 0 & 0 \\
\frac{1}{2 e^{2}} \delta(x-y) & 0 & \frac{1}{4 e^{2}} \epsilon(x-y)
\end{array}\right) .
$$

The reduced Hamiltonian density is obtained after the imposition of the constraints (23), (24) and (25) in Eq. (17) treating these constraints as strong conditions.

$\mathcal{H}_{R}=\frac{1}{2}\left(\pi_{1}^{2}+\phi^{\prime 2}\right)+\frac{1}{2 e^{2}} \pi_{1}^{\prime 2}-\pi_{1} A_{1}^{\prime}+\pi_{\phi} \phi^{\prime}+2 e^{2} A_{1}^{2}$.

From the definition (27), the Dirac brackets of the fields constituting the reduced Hamiltonian (31) are calculated as follows.

$$
\begin{aligned}
& {\left[A_{1}(x), A_{1}(y)\right]^{*}=-\frac{1}{2 e^{2}} \delta^{\prime}(x-y),} \\
& {\left[A_{1}(x), \pi_{1}(y)\right]^{*}=\delta(x-y),} \\
& {\left[A_{1}(x), \phi(y)\right]^{*}=-\frac{1}{2 e} \delta(x-y),} \\
& {\left[\pi_{1}(x), \pi_{1}(y)\right]^{*}=0,} \\
& {[\phi(x), \phi(y)]^{*}=\frac{1}{4} \epsilon(x-y) .}
\end{aligned}
$$

Here the superscript $(*)$ stands for indicating Dirac Bracket. The Hamiltonian (31) along with the Dirac brackets leads to the following three first order differential equations of motion

$\dot{\pi}_{1}=\pi_{1}^{\prime}-4 e^{2} A_{1}^{\prime}$,

$\dot{A}_{1}=\pi_{1}-A_{1}^{\prime}$,

$\dot{\phi}=-\phi^{\prime}-\frac{1}{e} \pi_{1}^{\prime}+2 e A_{1}$.

After a little algebra the above three equations render two second order and one first order differential equation which represent the theoretical spectra of the model.

$$
\begin{aligned}
& \left(\square+4 e^{2}\right) A_{1}=0, \\
& \left(\square+4 e^{2}\right) \pi_{1}=0, \\
& \partial_{+}\left[\phi+\frac{1}{2 e} \pi_{1}\right]=\partial_{+}\left[\phi+\frac{1}{2 e}\left(\dot{A}_{1}+A_{1}^{\prime}\right)\right]=0 .
\end{aligned}
$$

Here $\partial_{+}=\partial_{0}+\partial_{1}$. Note that all the these equations of motion are Lorentz invariant. So Physical Lorentz invariance is preserved in spite of the presence of the Lorentz non-covariant term in the bosonized action. The two second order Eqs. (40) and (41) correspond to the free massive boson and its momentum, respectively and Eq. (42) is representing a self dual (chiral) boson. The theoretical spectrum therefore contains a massive boson with mass $2 e$ and an massless chiral boson $[38,39]$. The extra degrees of freedom as predicted by Faddeev [32,33] is emerging here in the form of this very chiral boson. In $(1+1)$ dimension, it can be thought of in terms of a chiral fermion too. So the fermion which remains unconfined has a definite chirality. It is in contrast with the de-confinement scenario of chiral Schwinger model with Jackiw-Rajaraman type of regularization where a composite fermion having both the chiralities was unconfined. Now let us focus on the process of making a correlation which we have mentioned in Sect. 2.

We have seen in Sect. 2 that $\pi_{1}=-\epsilon_{\mu v}=\square F$. So we can immediately land on the conclusion that the field $\pi_{1}$ corresponds to $\square F$ which the massive free field as we have seen in both the Lagrangian and Hamiltonian formulation. Equations (10) and (42) show that $\mathcal{H}=\phi+\frac{1}{2 e} \pi_{1}$. So the spectrum obtained in the Lagrangian formulation and in the Hamiltonian formulation are in exact agreement with each other.

\section{Brief introduction of the BFV formalism}

BRST symmetry is the symmetry of a gauge fixed action in the enlarged Hilbert space. BRST quantization is subject of huge interest $[29,30,41-46,48-50]$. Field dependent BRST has added a new flavor in the arena of BRST quantization [51,52]. There are different formalism to get BRST invariant effective Lagrangian, however the combined formalism due to Batalin, Fradkin and Vilkovisky [41-43] for obtaining it is interesting and very effective in the context of having BRST invariant reformulation of a given model. It is based on the idea that a system with a set of second class constraints can be effectively converted to a first class set in the extended phase space which finally offers its service to construct BRST invariant effective action with the proper choice of gauge conditions. From the improved contribution of Fugiwara, Igarashi and Kubo (FIK) [44] in this regard we find that the field needed for this transmutation ultimately turns into the Wess-Zumino scalar. What follows next is a brief description of the general BFV formalism for obtaining a BRST invariant action.

If the Hamiltonian of a physical system is described by the canonical pairs $\left(p_{k}, q^{k}\right), k=1,2, \ldots, N$, where the canonical variables are endowed with a set of constraints $\Omega_{\alpha} \approx 0, \alpha=1,2 \ldots, N$, and if it is assumed that the constraints satisfy the following algebra

$\left[\Omega_{\alpha}, \Omega_{\beta}\right]=i \Omega_{\gamma} U_{\alpha \beta}^{\gamma}$, 
$\left[H_{c}, \Omega_{\alpha}\right]=i \Omega_{\beta} V_{\alpha}^{\beta}$,

then $N$ numbers of additional condition $\Phi_{\alpha} \approx 0$, with $\operatorname{det}\left[\Phi_{\alpha}, \Omega_{\beta}\right] \neq 0$ are beneficial to imposed into the phase space of the system in order to identify the physical degrees of freedom. At this state, the constraints $\Omega_{\alpha} \approx 0$ and $\Phi_{\alpha} \approx 0$, together with Hamiltonian equations of motion will be resulted out from the effective action

$S=\int d t\left[p_{k} \dot{q}^{k}-H_{c}\left(p_{k}, q^{k}\right)-\Lambda^{\alpha} \Omega_{\alpha}+\Pi_{\alpha} \Phi^{\alpha}\right]$,

where $\Lambda^{\alpha}$ and $\Pi_{\alpha}$ are Lagrange multiplier fields those which satisfy the canonical relation $\left[\Lambda^{\alpha}, \Pi_{\beta}\right]=i \delta^{\alpha_{\beta}}$.

Furthermore the launching of a pair of canonical ghost field $\left(C^{\alpha}, \bar{P}_{\alpha}\right)$ and a pair of canonical anti-ghost field $\left(P^{\alpha}, \bar{C}_{\alpha}\right)$ for each pair of constraints enables us to build up an equivalence between the initial theory with constraints in the reduced phase space. So the quantum theory can be described by the partition function $[41-43,45,46]$ which is holding the action

$$
\begin{aligned}
S_{G F}=\int & d t\left[p_{k} \dot{q}^{k}+\pi^{\alpha} \dot{\Lambda}_{\alpha}+\bar{P}^{\alpha} \dot{C}_{\alpha}\right. \\
& \left.+\bar{C}^{\alpha} \dot{P}_{\alpha}-H_{B R S T}+i[Q, \Gamma]\right],
\end{aligned}
$$

within its exponent. In Eq. (46), $H_{M}$ is the minimal Hamiltonian $[41,42]$ as termed by Batalin and Fradkin, is defined by

$H_{M}=H_{C}+\bar{P}_{\alpha} V_{\beta}^{\alpha} C^{\beta}$.

Consequently, the fermionic gauge fixing function $\Gamma$ is given by

$\Gamma=\bar{C}_{\alpha} \chi^{\alpha}+\bar{P}^{\alpha} \Lambda^{\alpha}$,

and the BRST charge $Q$ is by the expression

$Q=C^{\alpha} \Omega_{\alpha}-\frac{1}{2} C^{\alpha} C_{\gamma} U_{\alpha \beta}^{\gamma} \bar{P}^{\beta}+P^{\alpha} \Pi_{\alpha}$.

The gauge fixing conditions $\chi_{\alpha}$ 's are ultimately defined through expression

$\Phi_{\alpha}=\dot{\Lambda}_{\alpha}+\chi_{\alpha}$.

\section{Study BRST symmetry with the evolution of appropriate Wess-Zumino term}

Let us focus on the BRST invariant reformulation of the Lagrangian (5). In order to do that we need to know the constraint structure of the theory. The details of which is available in [5], but it is discussed in detail in Sect. 3 in a transparent manner for the shake of constancy and also for making this article self contained. Here we are giving the relevant portion as required for the present purpose.
We have seen that $\pi_{0} \approx 0$ is the primary constraint of the theory and the canonical Hamiltonian of the theory is

$$
\begin{aligned}
H_{C}=\int & d x\left[\frac{1}{2}\left(\pi_{1}^{2}+\pi_{\phi}^{2}+\phi^{\prime 2}\right)\right. \\
& \left.+\pi_{1} A_{0}^{\prime}-e\left(A_{0}-A_{1}\right)\left(\pi_{\phi}+\phi^{\prime}\right)+2 e^{2} A_{1}^{2}\right] .
\end{aligned}
$$

The full set of constraints are evaluated in Sect. 3. At a glance, the set of constraints those which are embedded in phase space of the theory are

$\omega_{1}=\pi_{0} \approx 0$,

$\omega_{2}=\pi_{1}^{\prime}+e\left(\pi_{\phi}+\phi^{\prime}\right) \approx 0$

$\omega_{3}=2 e^{2}\left(A_{0}+A_{1}\right) \approx 0$,

These three constraints form a second class set and the closures of the constrains with respect to the Hamiltonian (19) are found to be

$\dot{\omega}_{1}=\omega_{2}+\omega_{1}^{\prime}$,

$\dot{\omega}_{3}=\omega_{3}^{\prime}$,

$\dot{\omega}_{3}=-2 e^{2} \omega_{1}^{\prime}$.

According to the BVF prescription to wards BRST invariant reformulation, we now take an attempt to construct a first class set from the available set of second class constraints. We, therefore, introduce two pairs of canonical auxiliary fields $\eta, \pi_{\eta}$ and $\theta, \pi_{\theta}$ and insert the appropriate combination of these fields in the the second class set of constraint in such a manner that it can convert this second class set of constraints into first class set. These pair of fields are generally known as Batalin-Fradkin (BF) fields. The following are the converted first class constraints with the help of BF fields.

$\tilde{\omega}_{1}=\pi_{0}-\pi_{\eta}$

$\tilde{\omega}_{2}=\pi_{1}^{\prime}+e\left(\pi_{\phi}+\phi^{\prime}\right)-e\left(\pi_{\Psi}-\Psi^{\prime}-\pi_{\eta}^{\prime}\right)$,

$\tilde{\omega}_{3}=2 e^{2}\left(A_{0}+A_{1}\right)^{\prime}+2 e^{2} \eta^{\prime}$.

The above three first class constraints will be found consistent with the first class Hamiltonian (to be found out) if these new first class set follow the identical closures as their second class partner did with the Hamiltonian (19). Precisely, the conditions are

$\dot{\tilde{\omega}}_{1}=\tilde{\omega}_{2}+\tilde{\omega}_{1}^{\prime}$,
$\dot{\tilde{\omega}}_{2}=\tilde{\omega}_{3}^{\prime}$,
$\dot{\tilde{\omega}}_{3}=-2 e^{2} \tilde{\omega}_{1}^{\prime}$,

First class Hamiltonian is acquired by the appropriate placement of the BF fields within the Hamiltonian (19) which is given by $\tilde{H}=H_{C}+H_{B F}$. Here $H_{B F}$ is constituted with the polynomial of $\theta, \eta, \pi_{\theta}$ and $\pi_{\eta}$ that extend the phase space respecting the closures (61), (62) and (63). The first class 
Hamiltonian $H_{B F}$ ultimately results out to

$H_{B F}=\int d x\left[\frac{1}{2}\left(\pi_{\eta}^{2}+\pi_{\Psi}^{2}+\Psi^{\prime 2}\right)-e\left(\pi \Psi \eta-\eta \Psi^{\prime}\right)\right]$.

Three pairs of canonical ghost $\left(C_{i}, \bar{P}^{i}\right)$, and three pairs of canonical anti-ghost $\left(P_{i}, \bar{C}^{i}\right)$ fields along with four pairs of canonical multiplier fields $\left(N^{i}, B_{i}\right)$ are now brought into the system. The canonical relations for the fields are

$$
\begin{aligned}
{\left[C_{i}, \bar{P}^{j}\right] } & =\left[P^{i}, \bar{C}_{j}\right]=\left[N^{i}, B_{j}\right] \\
& =i \delta_{j}^{i} \delta(x-y), i=1,2,3
\end{aligned}
$$

In the present environment, we can write down BRST invariant Hamiltonian from the definition

$H_{U}=H_{M}-i[Q, \Gamma]$

where $H_{U}$ is the unitarizing Hamiltonian, $Q$ is the BRST charge and $\Gamma$ 's are the gauge fixing functions. To define BRST charge $Q$ nilpotency must be protected. The nilpotency is given by the condition

$Q^{2}=[Q, Q]=0$.

The definition of $Q$ in this formalism is

$Q=\int\left(B_{i} P^{i}+C_{i} \tilde{\omega}^{i}\right) d x$

and the definition of gauge fixing function $\Gamma$ is

$\Gamma=\int\left(\bar{C}_{i} \xi^{i}+P_{i} N^{i}\right) d x$,

where indices as usual take the values $i=1,2,3$. The BRST invariant Hamiltonian for this theory therefore can be expressed as

$$
\begin{aligned}
H_{U}= & H_{P}+H_{B F}+\int d x\left[\left(\pi_{0}-\pi_{\eta}\right) N^{1}\right. \\
& \left.+\left(\pi_{1}^{\prime}+e\left(\pi_{\phi}+\phi^{\prime}\right)-e\left(\pi_{\Psi}-\Psi^{\prime}\right)-\pi_{\eta}^{\prime}\right)\right) N^{2} \\
& +2 e^{2}\left(\left(A_{0}+A_{1}\right)^{\prime}-\eta^{\prime}\right) N^{3}-C^{1} \bar{C}_{1} \\
& -C^{2} \bar{C}_{2}^{\prime \prime}-2 e^{2} C_{3} \bar{C}_{3}^{\prime} \\
& -P^{1} \bar{P}-P^{2} \bar{P}_{2}-P^{3} \bar{P}_{3}+B_{1}\left(-\dot{N}_{2}-A_{0}\right) \\
& +B_{2}\left(\frac{B_{2}}{2}+A_{1}^{\prime}\right)+B_{3}\left(\pi_{\Psi}-\Psi^{\prime}\right) \\
& \left.+\left(P_{2}+P_{1}^{\prime}\right) C_{1}-2 e^{2} \bar{P}_{3}^{\prime} C_{2}-2 e^{2} \bar{P}_{1}^{\prime} C_{3}\right] .
\end{aligned}
$$

It would be helpful to write down the generating functional that ultimately leads to an effective action with the elimination of unwanted fields by Gaussian integration. The generating functional for the system now reads

$Z=\int[D \mu] e^{i S}$
Here the expression of $S$ :

$$
\begin{aligned}
S=\int & d^{2} x\left[\pi_{\phi} \dot{\phi}+\pi_{1} \dot{A}_{1}+\pi_{0} \dot{A}_{0}+\pi_{\Psi} \dot{\psi}+\pi_{\eta} \dot{\eta}\right. \\
& \left.+\bar{P}_{i} \dot{C}^{i}+\bar{C}_{i} \dot{P}^{i}+B_{i} \dot{N}^{i}-H_{U}\right]
\end{aligned}
$$

where $[D \mu]$ is the Liouville measure in the extended phase space which is given by

$$
\begin{gathered}
{[D \mu]=[d \phi]\left[d \pi_{\phi}\right] \sum_{i=0}^{1}\left[d A_{i}\right]\left[d \pi_{i}\right][d \eta]\left[d \pi_{\eta}\right][d \Psi]\left[d \pi_{\Psi}\right]} \\
\sum_{k=1}^{3}\left[d N^{k}\right]\left[d B_{k}\right]\left[d C^{k}\right]\left[d \bar{C}_{k}\right]\left[d P^{k}\right]\left[d \bar{P}_{k}\right] .
\end{gathered}
$$

We are now in a position to fix up the gauge conditions. We see that the following conditions will serve our purpose effectively.

$\xi_{1}=-\dot{N}_{2}+A_{0}$,

$\xi_{2}=\frac{B_{2}}{2}-A_{1}^{\prime}$,

$\xi_{3}=\pi_{\Psi}+\Psi^{\prime}$.

Our next task is now to simplify the action (1) through the elimination of undesired fields by Gaussian integration to reach to our desired result. A careful look reveals that here exists a simplification

$\int d^{2} x\left(B_{i} N^{i}+\bar{C}_{i} \dot{P}^{i}\right)=-i\left[Q, \int d^{2} x\left(\bar{C}_{i} \dot{N}^{i}\right)\right]$

with be Legendre transformation $B^{i} \rightarrow B^{i}+\dot{N}^{i}$. However, the simplification corresponding to $i=1$ only suffices in this situation. More simplification follows from the elimination of the fields $\pi_{0}, \pi_{1}, \pi_{\eta}, \pi_{p} h \mathrm{i}, \pi_{p}$ si $B_{1}, B_{3}, A_{0}, N^{1}$, $N^{3}, P_{1}, \bar{P}^{1}, P_{2}, \bar{P}^{2}, P_{1}, \bar{P}^{1}, C_{1}, \bar{C}^{1}, C_{3}$, and $\bar{C}^{3}$ through Gaussian integration with the absorption of the constant contribution of the integrals within the normalization. Ultimately, we obtain a very simplified generating functional that contains the following effective action within its exponent.

$$
\begin{aligned}
S_{e f f}= & \int d^{2} x\left[\frac{1}{2}\left(\dot{\phi}^{2}-\phi^{\prime 2}\right)+e\left(\dot{\phi}+\phi^{\prime}\right)\left(A_{0}-A_{1}\right)\right. \\
& +\frac{1}{2} e^{2}\left(A_{0}^{2}-2 A_{0} A_{1}-3 A_{1}^{2}\right) \\
& +\frac{1}{2}\left(\dot{A}_{1}-A_{0}^{\prime}\right)^{2} \\
& -\dot{\Psi} \Psi^{\prime}-\Psi^{\prime 2}-2 e \Psi^{\prime}\left(A_{1}+A_{0}\right)+\partial_{\mu} A^{\mu} \mathcal{B} \\
& \left.+\frac{1}{2} \alpha \mathcal{B}^{2}+\partial_{\mu} \bar{C} \partial_{\mu} C\right] .
\end{aligned}
$$

To get the simplified expression (78) we have used few redefinition of fields: $N_{2}=A_{0}$ and $P^{2}=\dot{C}_{2}$. Since after elimination there is no other $B$ 's and $C$ 's except $B_{2}$ and $C_{2}$ we are free to call these two as $\mathcal{B}$ and $C$, respectively. A little algebra shows that the action (78) is invariant under the BRST 
transformation

$\delta_{B} A_{1}=\lambda C^{\prime}, \delta_{B} A_{0}=\lambda \dot{C}, \delta_{B} \phi=\lambda C$,

$\delta_{B} \Psi=-\lambda C, \delta_{B} \bar{C}=\lambda \mathcal{B}, \delta_{B} C=0, \delta_{B} \mathcal{B}=0$.

At this stage we can easily recognize that the terms appearing in the action (78) which are constituted with the field $\Psi$ is the appropriate Wess-Zumino term [47] for this theory. It reads

$\mathcal{L}_{W Z}=-\dot{\Psi} \Psi^{\prime}-\Psi^{\prime 2}-2 e \Psi^{\prime}\left(A_{0}+A_{1}\right)$.

It is exiting to see this automatic emergence of this WessZumino term during the process BRST quantization. The role of gauge fixing is pivotal to get this appropriate WessZumino action. If it is not properly chosen one may fail to get appropriate Wess-Zumino term.

\section{Quantization of the gauge invariant action}

We now turn to the quantization of the gauge invariant action of this theory. The gauge symmetric version of the theory is no more than the original bosonized gauge no-invariant Lagrangian added with the Wess-Zumino term.

$\mathcal{L}_{G S}=\mathcal{L}_{G S}+\mathcal{L}_{W Z}$

To study the theoretical spectrum with this gauge symmetric version we need to fix the gauge redundancy of the theory $[22,23,53,54]$. In this context, we chose the Lorentz gauge, and the gauge fixing term with this gauge is

$\mathcal{L}_{G F}=\partial_{\mu} A^{\mu} \mathcal{B}+\frac{1}{2} \alpha \mathcal{B}^{2}$.

Therefore the Lagrangian with which we will set out for obtaining the theoretical spectrum is

$\mathcal{L}_{T O T}=\mathcal{L}_{G S}+\mathcal{L}_{G F}$

The Euler-Lagrange equations of motion that arise from the Lagrangian 83 are

$\square \phi+\left(\eta_{\mu \nu}+\epsilon_{\mu \nu}\right) \partial^{v} A^{\mu}=0$,

$\ddot{A}_{1}-\dot{A}_{0}^{\prime}+e\left(\dot{\phi}-\phi^{\prime}\right)+e^{2}\left(A_{0}+3 A_{1}\right)=0$,

$\dot{A}_{1}^{\prime}-A_{0}^{\prime \prime}+e\left(\dot{\phi}-\phi^{\prime}\right)+e^{2}\left(A_{0}-A_{1}\right)=0$,

$\dot{\Psi}^{\prime}-\Psi^{\prime \prime}-e\left(A_{0}+A_{1}\right)^{\prime}=0$.

These set of coupled equations get decoupled if the following ansatz are taken for the fields $A_{\mu}, \phi$ and $\Psi$.

$$
\begin{aligned}
& A_{\mu}=\frac{1}{2 e^{2}} \tilde{\partial}_{\mu} \mathcal{F}+\partial_{\mu} \mathcal{B}+\tilde{\partial}_{\mu} \mathcal{H}-\partial_{\mu} \mathcal{K}, \\
& \phi=-\frac{1}{2 e} \mathcal{F}+\mathcal{K}-\mathcal{H}+\mathcal{B}, \\
& \Psi=-\frac{1}{2 e} \mathcal{F}-\mathcal{K}-\mathcal{H}+\mathcal{B} .
\end{aligned}
$$

With the above ansatz, we have the simplification as follows

$$
\begin{aligned}
& \left(\square+4 e^{2}\right) \square \mathcal{F}=0, \\
& \partial_{-} \mathcal{H}=0, \\
& \square \mathcal{B}=0, \\
& \square \mathcal{K}-e \alpha \mathcal{B}=0 .
\end{aligned}
$$

Therefore the system is described by four free fields $\mathcal{F}, \mathcal{H}, \mathcal{B}$, and $\mathcal{K}$. Our next task is to express these four fields in terms of the field with which the theory under investigation is defined. After a few steps of algebra we find that

$\square \mathcal{F} \approx \mathcal{F}=\pi_{1}$

$\mathcal{H}=\phi+\frac{1}{e} \pi$,

$\mathcal{B}=\pi_{0}$

$\mathcal{K}=\frac{1}{2}(\Phi-\Psi)$.

Therefore the field $\mathcal{F}=\pi$ corresponds to a massive field with mass $2 e$, and the field $\mathcal{H}$ corresponds to a massless chiral boson. These two fields replicates the spectrum as obtained in Sect. 3. The equations involving $\mathcal{B}$ enters into picture because Lorentz gauge fixing term holds the auxiliary field $\mathcal{B}$. Note that the field $\mathcal{B}$ and $\mathcal{K}$ give vanishing commutation with the physical field $\pi$ and $\mathcal{H}$. So these two fields do not have any influence on the physical sector of the theory. The field $\mathcal{K}$ stands for the zero mass dipole field playing the role of gauge degrees of freedom which can be readily eliminated by operator gauge transformation. So the theoretical spectra are in exact agreement with the theoretical spectra obtained in [5] in the Hamiltonian formulation.

\section{Mapping of the gauge symmetric action onto the usual gauge non-invariant action}

Equation (78) is the BRST invariant effective action corresponding to the chiral Schwinger model with Faddeevian anomaly. The $\mathrm{U}(1)$ gauge symmetric part of this action is

$$
\begin{aligned}
S_{G S E A}= & \int d^{2} x\left[\frac{1}{2}\left(\dot{\phi}^{2}-\phi^{\prime 2}\right)+e\left(\dot{\phi}+\phi^{\prime}\right)\left(A_{0}-A_{1}\right)\right. \\
& +\frac{1}{2} e^{2}\left(A_{0}^{2}-2 A_{0} A_{1}-3 A_{1}^{2}\right) \\
& +\frac{1}{2}\left(\dot{A_{1}}-A_{0}^{\prime}\right)^{2}+\frac{1}{2}\left(\dot{A_{1}}-A_{0}^{\prime}\right)^{2} \\
& \left.-\dot{\Psi} \Psi^{\prime}-\Psi^{\prime 2}-2 e \Psi^{\prime}\left(A_{1}+A_{0}\right)\right] .
\end{aligned}
$$

It is the usual action of this theory along with the WessZumino action as developed in Sect. 4. during the BRST invariant reformulation of this model. It would be instructive to show that this gauge symmetric action is equivalent to the gauge non-invariant action. In other words, the physical 
contents of both the gauge invariant and gauge non-invariant action is identical, and the Wess-Zumino field has no impact
From the above inputs we can straight away write down the the generating functional.

$$
Z=\int[\mathcal{D} \mu]\left[\operatorname{det}\left[\mathcal{C}_{m}, \mathcal{C}_{n}\right]\right]^{\frac{1}{2}} e^{i \int d^{2} x\left[\pi_{1} \dot{A}_{1}+\pi_{0} \dot{A}_{0}+\pi_{\phi} \dot{\phi}+\pi_{\theta} \dot{\theta}-H_{C G S}\right]} \delta\left(\mathcal{C}_{1}\right)\left(\mathcal{C}_{2}\right) \delta\left(\mathcal{C}_{3}\right) \delta\left(\mathcal{C}_{4}\right) \delta\left(\mathcal{C}_{5}\right)
$$

to the physical sector of the theory. To make the equivalence appropriate gauge fixing is needed which we have learned from the edifying article [55]. So we have chosen gauge condition which will be well suited to project this gauge symmetric action onto the gauge non-invariant action. We will follow the path integral formulation in this regard with that befitting gauge.

To proceed with this purpose we need the momenta corresponding to the fields $\phi, A_{0}, A_{1}$ and $\Psi$ those which are obtained from the action (99):

$$
\begin{aligned}
& \frac{\partial S_{G S E A}}{\partial \dot{A}_{0}}=\pi_{0} \approx 0, \\
& \frac{\partial S_{G S E A}}{\partial \dot{A}_{1}}=\pi_{1}=\left(\dot{A}_{1}-A_{0}^{\prime}\right), \\
& \frac{\partial S_{G S E A}}{\partial \dot{\phi}}=\pi_{\phi}=\dot{\phi}+e\left(A_{0}-A_{1}\right), \\
& \frac{\partial S_{G S E A}}{\partial \dot{\Psi}}=\pi_{\Psi}=-\psi^{\prime} .
\end{aligned}
$$

The Eqs. (100) and (103) are independent of velocities so these two equations represent the primary constraints of the gauge symmetric action. Explicitly, these two are

$\mathcal{C}_{1}=\pi_{0} \approx 0$,

$\mathcal{C}_{2}=\pi_{\Psi}+\Psi^{\prime} \approx 0$.

The Eqs. (100), (101), (102) and (103), along with a Legendre transformation lead to the canonical Hamiltonian $H_{C G S}$ :

$$
\begin{aligned}
H_{C G S}= & \int d x\left[\frac{1}{2}\left(\pi_{1}^{2}+\pi_{\phi}^{2}+\phi^{\prime 2}\right)+\pi_{1} A_{0}^{\prime}-e\left(\pi_{\phi}+\phi^{\prime}\right)\left(A_{0}-A_{1}\right)\right. \\
& \left.+2 e^{2} A_{1}^{2}+\Psi^{\prime 2}+2 e\left(A_{0}+A_{1}\right) \Psi^{\prime}\right] .
\end{aligned}
$$

The preservation of the constraint of $\mathcal{C}_{1}$ provides with a new constraint

$\left.\mathcal{C}_{3}=\pi_{1}^{\prime}+e\left(\pi_{\phi}+\phi^{\prime}\right)-2 e \Psi^{\prime}\right) \approx 0$.

The articles [55,56], render necessary insight for choosing legitimate gauge to make the equivalence between the gauge symmetric and gauge nonsymmetric theories. The conditions those which have been found beneficial to make this equivalence in the present situation are

$\mathcal{C}_{4}=\Psi^{\prime} \approx 0$,
$\mathcal{C}_{5}=\pi_{\psi}+e\left(A_{0}+A_{1}\right) \approx 0$. where the Liouville measure $[\mathcal{D} \mu]=d \pi_{\phi} d \phi d \pi_{1} d A_{1} d \pi_{0} d$ $A_{0} d \pi_{\theta} d \theta$, and $m$ and $n$ run from 1 to 5 . After integrating out of the field $\Psi$ and $\pi_{\Psi}$, we find that the generating functional turns into

$$
\begin{aligned}
Z=N & \int[d \bar{\mu}] e^{i \int d^{2} x\left[\pi_{1} \dot{A}_{1}+\pi_{0} \dot{A}_{0}+\pi_{\phi} \dot{\phi}+-\tilde{H}_{C N S} \bar{H}_{C}\right]} \\
& \times \delta\left(\pi_{0}\right) \delta\left(\pi_{1}^{\prime}+e\left(\pi_{\phi}+\phi^{\prime}\right) \delta\left(A_{0}+A_{1}\right),\right.
\end{aligned}
$$

where $[\mathcal{D} \tilde{\mu}]=d \pi_{\phi} d \phi d \pi_{1} d A_{1} d \pi_{0} d A_{0}$ and $N$ is a normalization constant having no significant physical importance, and $\tilde{H}_{C N S}$ is given by

$$
\begin{aligned}
\tilde{H}_{C N S}= & \frac{1}{2}\left(\pi_{1}^{2}+\phi^{\prime 2}+\pi_{\phi}^{2}\right)+\pi_{1} A_{0}^{\prime} \\
& +e\left(\pi_{\phi}+\phi^{\prime}\right)\left(A_{0}-A_{1}\right)+2 e A_{1}^{2} .
\end{aligned}
$$

We land onto the required result after integrating out of the momenta $\pi_{\phi}, \pi_{1}$ and $\pi_{0}$ :

$Z=\int d A_{1} d A_{0} d \phi e^{i \mathcal{S}_{C B}}$

So the theory of the gauge symmetric action in extended phase space and the gauge non-symmetric action in the usual phase space are equivalent. So it is evident that the physical sector remain unperturbed in presence of the Wess-Zumino field. It will be more transparent if we look carefully to the Dirac Brackets of the fields. We, therefore, proceed to compute the Dirac brackets of the fields using the definition (27). The matrix $C_{i j}$ which is constituted with Poisson bracket among the constraints with each other is

$C_{i j}=\left(\begin{array}{ccccc}0 & 0 & 0 & 0 & -e \delta(x-y) \\ 0 & -2 \delta^{\prime}(x-y) & 2 e \delta^{\prime}(x-y) & \delta^{\prime}(x-y) & \delta^{\prime}(x-y) \\ 0 & 2 e \delta^{\prime}(x-y) & 2 e \delta^{\prime}(x-y) & 0 & e \delta^{\prime}(x-y) \\ 0 & \delta^{\prime}(x-y) & 0 & 0 & \delta(x-y)^{\prime} \\ e \delta(x-y) & \delta^{\prime}(x-y) & 2 \delta^{\prime}(x-y) & \delta^{\prime}(x-y) & 0\end{array}\right)$.

Using the definition (27), it is kids' stuff to compute the Dirac brackets between the different fields and their momenta:

$$
\begin{aligned}
& {\left[A_{1}(x), A_{1}(y)\right]^{*}=\frac{1}{e^{2}} \delta^{\prime}(x-y),} \\
& {\left[A_{1}(x), \pi_{1}(y)\right]^{*}=\delta(x-y),} \\
& {\left[A_{1}(x), \phi(y)\right]^{*}=-\frac{1}{2 e} \delta(x-y),} \\
& {\left[\pi_{1}(x), \pi_{1}(y)\right]^{*}=0,} \\
& {[\phi(x), \phi(y)]^{*}=-\frac{1}{4} \epsilon(x-y),}
\end{aligned}
$$


$\left[\phi(x), \pi_{\phi}(y)\right]^{*}=\delta(x-y)$,

$\left[A_{0}(x), \phi(y)\right]^{*}=\frac{1}{e} \delta(x-y)$.

The superscript $(*)$ in this case symbolizes the Dirac bracket. The Dirac Brackets computed here are found to be identical with the Dirac Brackets computed in Sect. 3. The Dirac bracts of the Wess-Zumino field and its canonical momenta give vanishing contribution with the physical field. This indicates that the physical fields remains unperturbed in presence of the Wess-Zumino fields. In fact, the Wess-Zumino field allocates themselves in the un-physical sector of the theory.

\section{Summary and discussion}

In this article we have considered the chiral Schwinger model with Faddeevian anomaly coined by Mitra in his seminal article [5]. The theoretical spectrum of this gauge non-invariant model has been determined here using Lagrangian as well as Hamitonian formulation. The spectra come in exact agreement with the spectra determined by Mitra in [5].

The BRST quantization has been carried out using BVF based improved formulation by FIK. It is exiting to mention that during the process of BRST quantization Wess-Zumino term has evolved out spontaneously. However it should be kept in mind that gauge fixing plays a crucial role in order to obtain the appropriate Wess-Zumino term.

Quantization of the gauge invariant version of this model also has been carried out in this article in the Lagrangian formulation with Lorentz gauge. How the fields get correlated with physical fields that describe the usual gauge non-invariant Lagrangian has been clearly explored. The unphysical fields has been found to have no influence to the physical sector since these fields have vanishing Poisson bracket with the physical fields. The auxiliary (un-physical) fields appearing in the gauge symmetric version allocate themselves in the un-physical sector of the theory.

An attempt has been made to show how the Gauge invariant version gets mapped onto the gauge non-invariant version with the proper choice of gauge fixing condition. Gauge fixing has a pivotal role for this transmutation too.

Acknowledgements I would like to thanks the Director Saha Institute of Nuclear Physics, Kolkata, for permitting me to use the and computer and library facilities of the Institute.

Data Availability Statement This manuscript has no associated data or the data will not be deposited. [Authors' comment: It is an analytical field theoretical article. So no data is needed.]

Open Access This article is licensed under a Creative Commons Attribution 4.0 International License, which permits use, sharing, adaptation, distribution and reproduction in any medium or format, as long as you give appropriate credit to the original author(s) and the source, provide a link to the Creative Commons licence, and indicate if changes were made. The images or other third party material in this article are included in the article's Creative Commons licence, unless indicated otherwise in a credit line to the material. If material is not included in the article's Creative Commons licence and your intended use is not permitted by statutory regulation or exceeds the permitted use, you will need to obtain permission directly from the copyright holder. To view a copy of this licence, visit http://creativecomm ons.org/licenses/by/4.0/.

Funded by SCOAP ${ }^{3}$.

\section{References}

1. R. Jackiw, R. Rajaraman, Phys. Rev. Lett. 54, 1219 (1985)

2. H.O. Girotti, H.J. Rothe, K.D. Rothe, Phys. Rev. D 33, 514 (1986)

3. H.O. Girotti, H.J. Rothe, K.D. Rothe, Phys. Rev. D 34, 592 (1986)

4. K. Harada, Phys. Rev. Lett. 64, 139 (1990)

5. P. Mitra, Phys. Lett. B 284, 23 (1992)

6. J. Schwinger, Phys. Rev 128, 2425 (1962)

7. J.H. Lowenstein, J.A. Swieca, Ann. Phys. (N. Y.) 68, 172 (1971)

8. C.R. Hagen, Ann. Phys. (N. Y.) 81, 67 (1973)

9. R. Banerjee, Phys. Rev. Lett. 56, 1986 (1889)

10. S. Ghosh, P. Mitra, Phys. Rev. D 44, 1332 (1990)

11. S. Mukhopadhyay, P. Mitra, Zeit. f. Phys. C 97, 552 (1995)

12. S. Mukhopadhyay, P. Mitra, Ann. Phys. (N. Y.) 241, C68 (1995)

13. A. Ghosh, P. Mitra, Phys. Rev. D 50, 7389 (1994)

14. P. Mitra, A. Rahaman, Ann. Phys. (N. Y.) 249, 34 (1996)

15. A. Rahaman, Int. J. Mod. Phys. A 12, 5625 (1997)

16. A. Rahaman, Int. J. Mod. Phys. A 19, 3013 (2004)

17. A. Rahaman, Int. J. Mod. Phys. A 21, 1251 (2006)

18. A. Rahaman, Mod. Phys. Lett. A 24, 2195 (2009)

19. A. Rahaman, Phys. Lett. B 697, 260 (2011)

20. A. Rahaman, Nucl. Phys. B 932, 119 (2018)

21. A. Rahaman, Ann. Phys. (N.Y.) 361, 33 (2015)

22. A. Rahaman, Ann. Phys. (N.Y.) 354, 511 (2015)

23. A. Rahaman, S. Yasmin, Ann. Phys. 383, 497 (2017)

24. P. Mitra, R. Rajaraman, Ann. Phys. (N. Y.) 203, 137 (1990)

25. P. Mitra, R. Rajaraman, Ann. Phys. (N. Y.) 203, 157 (1990)

26. A. Rahaman, S. Yasmin, S. Aziz, Int. J. Theor. Phys. 49, 2607 (2010)

27. V. Domcke, Y. Ema, K. Mukaida, R. Sato JHEP 1903, 111 (2019)

28. B. Buyens, F. Verstraete, K.V. Acoleyen, Phys. Rev. D 94, 085018 (2016)

29. Y.W. Kim, S.K. Kim, W.T. Kim, Y.J. Park, K.Y. Kim, Y. Kim, Phys. Rev. D 46, 4574 (1992)

30. S. Ghosh, Phys. Rev. D 49, 2990 (1994)

31. Y.G. Miao, J.G. Zhou, Y.Y. Liu, J. Phys. G 19, 1797 (1993)

32. L.D. Faddeev, Phys. Lett. B 154, 81 (1984)

33. L.D. Faddeev, S.L. Shatashvili, Phys. Lett. B 167, 225 (1986)

34. S.L. Shatashvili, Theor. Math. Phys. 60, 770 (1985)

35. S.L. Shatashvili, Theor. Mat. Fiz. 60, 206 (1984)

36. W. Siegel, Nucl. Phys. B 238, 307 (1984)

37. J. Sonnenschein, Nucl. Phys. B 309, 752 (1988)

38. S. Bellucci, M.F.L. Golterman, D.N. Petcher, Nucl. Phys. B 326, 307 (1989)

39. R. Floreanini, R. Jackiw, Phys. Rev. Lett. 59, 1873 (1987)

40. P.A.M. Dirac, Lectures on quantum Mechanics (Yeshiva Univ. Press, New York, 1964)

41. E.S. Fradkin, G.A. Vilkovisky, Phys. Lett. B 55, 224 (1975)

42. I.A. Batalin, E.S. Fradkin, Nucl. Phys. B 279, 514 (1987)

43. I.A. Batalin, V. Tyutin, Int. J. Mod. Phys. A 6, 3255 (1991)

44. T. Fujiwara, I. Igarashi, J. Kubo, Nucl. Phys. B 314, 695 (1990)

45. S.J. Yoon, Y.W. Kim, Y.J. Park, J. Phys. G 25, 1783 (1989)

46. M.I. Park, Y.J. Park, S.J. Yoon, J. Phys. G 24, 2179 (1988)

47. J. Wess, B. Zumino, Phys. lett. B 37, 95 (1971) 
48. T. Bhanja, R.P. Malik, Eur. Phys. J. C 74, 3025 (2014)

49. A. Rahaman, S. Yasmin, Ann. Phys. (N.Y.) 383, 497 (2017)

50. R.P. Malik, Eur. Phys. J. C 47, 227 (2006)

51. S. Upadhyay, B.P. Mandal, Eur. Phys. J. C 72, 2065 (2012)

52. S. Upadhyay, B.P. Mandal, Eur. Phys. J. C 71, 1759 (2011)

53. S. Miyake, K. Shizuya, Phys. Rev. D 36, 3781 (1987)
54. S. Miyake, K. Shizuya, Phys. Rev. D 37, 2288 (1988)

55. N.C. Falck, G. Kramer, Ann. Phys. (N. Y.) 176, 330 (1987)

56. S. Yasmin, A. Rahaman, Int. Jour. Mod. Phys. A 310, 1650171 (2016) 